\title{
INDECOMPOSABLE EXPOSED POSITIVE BI-LINEAR MAPS BETWEEN TWO BY TWO MATRICES
}

\author{
SEUNG-HYEOK KYE
}

\begin{abstract}
Positive bi-linear maps between matrix algebras play important roles to detect tri-partite entanglement by the duality between bi-linear maps and tri-tensor products. We exhibit indecomposable positive bi-linear maps between $2 \times 2$ matrices which generate extreme rays in the cone of all positive bi-linear maps. In fact, they are exposed, and so detect entanglement of positive partial transpose whose volume is nonzero.
\end{abstract}

\section{InTRODUCTION}

Positive linear maps in matrix algebras are indispensable to distinguish entanglement from separability, by the duality [8, 16] between bi-partite separable states and positive linear maps. The convex cone consisting of positive linear maps is very complicated even in the cases when the domains and ranges are low dimensional matrices, and its whole structures are far from to be understood. Typical examples of positive maps from $m \times m$ matrices into $n \times n$ matrices are of the forms

$$
X \mapsto V^{*} X V, \quad X \mapsto W^{*} X^{\mathrm{t}} W
$$

with the transpose $X^{\mathrm{t}}$ of $X$ and $m \times n$ matrices $V$ and $W$. A sum of the first (respectively second) type is called completely positive (respectively completely copositive), and a sum of them is said to be decomposable. It is known that every positive map from $M_{m}$ into $M_{n}$ is decomposable if and only if $(m, n)$ is $(2,2),(2,3)$ or $(3,2)$ [4, 25, 26], and many examples of indecomposable positive linear maps are known. See the survey article [18].

Recall that a positive map is said to be exposed (respectively extremal) if it generates an exposed (respectively extreme) ray in the convex cone of all positive linear maps. Among various examples of indecomposable positive linear maps, exposed maps attract special interests in quantum information theory. First of all, indecomposable exposed maps detect PPT entanglement of nonzero volume. Furthermore, exposed maps are enough to determine separability, by the classical Straszewicz's Theorem: Exposed points make a dense subset in the set of all extreme points. See [23], Theorem 18.6. In case of decomposable maps, the maps in (11) are known to be exposed [20, 28], and there are no other exposed decomposable maps.

1991 Mathematics Subject Classification. 46L07, 15A30, 81P15.

Key words and phrases. positive multi-linear maps, exposed, indecomposable, separable, partial transpose.

partially supported by NRF-2017R1A2B4006655. 
Nevertheless, there are very few known examples of exposed indecomposable positive linear maps in the literature. Some variants [2] of the Choi map [5] between $M_{3}$ had been proved to be exposed in [9]. Note that the Choi map itself is not exposed, even though it is extremal. On the other hand, the Robertson's map [21, 22] between $M_{4}$ was shown [7] to be exposed. Some variants in the higher dimensional cases have been also considered in [24]. As for linear maps between $M_{2}$ and $M_{4}$, a large class of indecomposable exposed positive linear maps have been constructed in [11], motivated by the example of Woronowicz [27].

The author [19] had considered multi-partite version of the duality, and exhibited examples of indecomposable positive bi-linear maps with the full spanning property between $2 \times 2$ matrices, in order to get examples of three qubit separable states with full ranks. A positive map with the full spanning property is automatically indecomposable, and indecomposable exposed positive maps must have the spanning property. See the next section for the details. Nevertheless, the spanning property does not imply the exposedness in general, even in the bi-partite systems. See the example in Section 5 of [10].

The purpose of this note is to show that the positive bi-linear maps constructed in [19] are, in fact, exposed. The main tool is to use the four dimensional norm introduced in [1]. This norm turns out to be crucial to characterize [1] separability of an important class of three qubit states, like Greenberger-Horne-Zeilinger diagonal states. See also [14, 15]. These provide the first concrete examples of indecomposable exposed positive bi-linear maps.

In the next section, we review very briefly the duality between multi-partite separability and positivity of multi-linear maps, and explain the motivation of the construction in [19]. In section 3, we compute the dual and the double dual to show that the map is exposed.

The author is grateful to Kyung-Hoon Han for useful discussion and comments.

\section{Duality And indecomposable Positive Bi-Linear maps}

For a given multi-linear map $\phi: M_{d_{1}} \times \cdots \times M_{d_{n-1}} \rightarrow M_{d_{n}}$, we associate a matrix $C_{\phi} \in M_{d}$ with $d=d_{1} d_{2} \cdots d_{n}$ by

$$
C_{\phi}=\sum_{i_{1}, j_{1}, \ldots, i_{n-1}, j_{n-1}}\left|i_{1}\right\rangle\left\langle j_{1}|\otimes \cdots \otimes| i_{n-1}\right\rangle\left\langle j_{n-1}\right| \otimes \phi\left(\left|i_{1}\right\rangle\left\langle j_{1}|, \cdots,| i_{n-1}\right\rangle\left\langle j_{n-1}\right|\right) .
$$

The matrix $C_{\phi}$ is call the Choi matrix of $\phi$. The correspondence $\phi \mapsto C_{\phi}$ is just the Choi-Jamiołkowski isomorphism [3, 17] for bi-partite case of $n=2$. See also [6] for multi-partite cases. For a state $\varrho \in M_{d}$ and an $(n-1)$-linear map $\phi$, we consider the bilinear pairing $\langle\varrho, \phi\rangle$ defined by

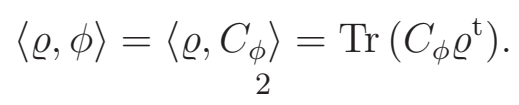


Recall that a state in $M_{d}$ is said to be separable if it is the convex combination of pure product states, and a multi-linear map $\phi: M_{d_{1}} \times \cdots \times M_{d_{n-1}} \rightarrow M_{d_{n}}$ is called positive if $\phi\left(x_{1}, \ldots x_{n-1}\right) \in M_{d_{n}}$ is positive whenever $x_{i} \in M_{d_{i}}$ is positive for every $i=1,2, \ldots, n-1$. If $\varrho=|\xi\rangle\langle\xi|$ is a pure product state with the product vector $|\xi\rangle=\left|\xi_{1}\right\rangle \cdots\left|\xi_{n}\right\rangle$ then we have the relation

$$
\langle\varrho, \phi\rangle=\left\langle C_{\phi}, \mid \xi\right\rangle\langle\xi \mid\rangle=\left\langle\bar{\xi}\left|C_{\phi}\right| \bar{\xi}\right\rangle=\left\langle\bar{\xi}_{n}\left|\phi\left(\left|\xi_{1}\right\rangle\left\langle\xi_{1}|, \ldots,| \xi_{n-1}\right\rangle\left\langle\xi_{n-1}\right|\right)\right| \bar{\xi}_{n}\right\rangle .
$$

Therefore, we see [19] that a multi-linear map $\phi$ is positive if and only if $\langle\varrho, \phi\rangle \geq 0$ for every separable state $\varrho$. By the Hahn-Banach type separation theorem, we see that $\varrho$ is separable if and only if $\langle\varrho, \phi\rangle \geq 0$ for every positive multi-linear map $\phi$. We also see that a self-adjoint matrix $W$ is the Choi matrix of a positive multi-linear map if and only if $\langle\xi|W| \xi\rangle \geq 0$ for every product vector $|\xi\rangle$. Those self-adjoint matrices are called block-positive. We say that a multi-linear map $\phi$ is completely positive if its Choi matrix $C_{\phi}$ is positive (semi-definite). See [3] for linear cases.

For a given positive multi-linear map $\phi$, the dual $\phi^{\prime}$ of $\phi$ is defined by the set of all (unnormalized) separable states $\varrho$ such that $\langle\varrho, \phi\rangle=0$. The set $\phi^{\prime}$ is an exposed face of the convex cone of all separable (unnormalized) states. We denote by $P[\phi]$ the set of all product vectors $|\xi\rangle$ such that $\left\langle\xi\left|C_{\phi}\right| \xi\right\rangle=0$. The double dual $\phi^{\prime \prime}$ is also defined by the set of all positive maps $\psi$ satisfying $\langle\varrho, \psi\rangle=0$ for every $\varrho \in \phi^{\prime}$. The double dual $\phi^{\prime \prime}$ is the smallest exposed face containing the map $\phi$, and so we see that $\phi$ is exposed if and only if $\phi^{\prime \prime}$ consists of nonnegative multiples of $\phi$.

For a given subset $S$ of $\{1,2, \ldots, n\}$, the partial transpose $A^{T(S)}$ is defined by

$$
\left(A_{1} \otimes A_{2} \otimes \cdots \otimes A_{n}\right)^{T(S)}:=B_{1} \otimes B_{2} \otimes \cdots \otimes B_{n}, \quad \text { with } B_{j}= \begin{cases}A_{j}^{\mathrm{t}}, & j \in S, \\ A_{j}, & j \notin S,\end{cases}
$$

for multi-partite matrices. A state $\varrho$ is said to be of PPT (positive partial transpose) if $\varrho^{T(S)}$ is positive for every subset $S$. On the other hand, $\phi$ is called decomposable if its Choi matrix is the sum of partial transposes of positive matrices. It is clear that $\phi$ is decomposable if and only if $\langle\varrho, \phi\rangle \geq 0$ for every PPT state $\varrho$, and $\varrho$ is of PPT if and only if $\langle\varrho, \phi\rangle \geq 0$ for every decomposable map $\phi$. On the other hand, the partial conjugate $|\xi\rangle^{\Gamma(S)}$ is defined by

$$
\left(\left|\xi_{1}\right\rangle \otimes \cdots \otimes\left|\xi_{n}\right\rangle\right)^{\Gamma(S)}:=\left|\eta_{1}\right\rangle \otimes \cdots \otimes\left|\eta_{n}\right\rangle, \quad \text { with }\left|\eta_{j}\right\rangle= \begin{cases}\left|\bar{\xi}_{j}\right\rangle, & j \in S, \\ \left|\xi_{j}\right\rangle, & j \notin S,\end{cases}
$$

for product vectors. We say that $\phi$ has the full spanning property if the set $\left\{|\xi\rangle^{\Gamma(S)}\right.$ : $\xi \in P[\phi]\}$ spans the whole space for every $S$. It was shown in [19] that the following are equivalent for a positive multi-linear map $\phi$ :

- $\phi$ has the full spanning property.

- The interior of the face $\phi^{\prime}$ lies in the interior of convex cone of all PPT states.

- The smallest exposed face $\phi^{\prime \prime}$ containing $\phi$ has no decomposable map.

- The set of PPT states $\varrho$ satisfying $\langle\varrho, \phi\rangle<0$ has a nonempty interior. 
The last statement tells us that the map $\phi$ detects PPT entanglement with nonzero volume. By the third condition, we also see that every indecomposable exposed map satisfies the above properties.

Now, we proceed to construct an example of indecomposable positive bi-linear map $\phi: M_{2} \times M_{2} \rightarrow M_{2}$. It is a positive bi-linear map if and only if the corresponding linear map $M_{2} \rightarrow \mathcal{L}\left(M_{2}, M_{2}\right)$ sends positive matrices into positive maps if and only if it sends positive matrices into decomposable maps by [25] if and only if the corresponding linear map $M_{2} \rightarrow M_{4}$ sends positive matrices into matrices which are sums of positive matrices and co-positive matrices, that is, partial transposes of positive matrices. In other words, it is the sum of two maps $\phi_{1}$ and $\phi_{2}$, where $\phi_{1}$ and $\phi_{2}$ send positive matrices into positive and co-positive matrices, respectively. If both of $\phi_{1}$ and $\phi_{2}$ are linear then the corresponding bi-linear map $\phi$ must be decomposable. Therefore, we have to look for non-linear maps $\phi_{1}$ and $\phi_{2}$ with the above properties so that $\phi_{1}+\phi_{2}$ is linear. For every complex numbers, we consider the rank one matrix $P_{\alpha}=\left(\begin{array}{cc}1 & \bar{\alpha} \\ \alpha & |\alpha|^{2}\end{array}\right)$, and put

$$
A_{\alpha}=\left(\begin{array}{cccc}
\cdot & \cdot & \cdot & \cdot \\
\cdot & |\bar{\alpha}+\alpha|^{2} & \bar{\alpha}+\alpha & \cdot \\
\cdot & \alpha+\bar{\alpha} & 1 & \cdot \\
\cdot & \cdot & \cdot & \cdot
\end{array}\right), \quad B_{\alpha}=\left(\begin{array}{cccc}
\cdot & \cdot & \cdot & \cdot \\
\cdot & |\bar{\alpha}-\alpha|^{2} & \bar{\alpha}-\alpha & \cdot \\
\cdot \alpha-\bar{\alpha} & 1 & \cdot \\
\cdot & \cdot & \cdot
\end{array}\right)
$$

where $\cdot$ denotes zero. Neither $P_{\alpha} \mapsto A_{\alpha}$ nor $P_{\alpha} \mapsto B_{\alpha}^{T}$ are linear. But, we note that the map

$$
P_{\alpha} \mapsto A_{\alpha}+B_{\alpha}^{T}=\left(\begin{array}{cccc}
\cdot & \cdot & \cdot & \bar{\alpha}-\alpha \\
\cdot & 4|\alpha|^{2} & \bar{\alpha}+\alpha & \cdot \\
\cdot & \bar{\alpha}+\alpha & 2 & \cdot \\
\alpha-\bar{\alpha} & \cdot & \cdot & \cdot
\end{array}\right)
$$

is linear.

Motivated by the above discussion, we have considered in [19] the bilinear map $\phi: M_{2} \times M_{2} \rightarrow M_{2}$ which sends $\left(\left[x_{i j}\right],\left[y_{i j}\right]\right) \in M_{2} \times M_{2}$ to

$$
\left(\begin{array}{cc}
s x_{22} y_{11} & x_{12} y_{12}-x_{12} y_{21}+x_{21} y_{12}+x_{21} y_{21} \\
x_{12} y_{12}+x_{12} y_{21}-x_{21} y_{12}+x_{21} y_{21} & t x_{11} y_{22}
\end{array}\right) \in M_{2},
$$

where $s, t$ are positive numbers with $\sqrt{s t}=2 \sqrt{2}$. Note that the corresponding Choi matrix is given by

$$
C_{\phi}=\left(\begin{array}{cccccccc}
\cdot & \cdot & \cdot & \cdot & \cdot & \cdot & \cdot & 1 \\
\cdot & \cdot & \cdot & \cdot & \cdot & \cdot & 1 & \cdot \\
\cdot & \cdot & \cdot & \cdot & \cdot & -1 & \cdot & \cdot \\
\cdot & \cdot & \cdot & t & 1 & \cdot & \cdot & \cdot \\
\cdot & \cdot & \cdot & 1 & s & \cdot & \cdot & \cdot \\
\cdot & \cdot & -1 & \cdot & \cdot & \cdot & \cdot & \cdot \\
\cdot & 1 & \cdot & \cdot & \cdot & \cdot & \cdot & \cdot \\
1 & \cdot & \cdot & \cdot & \cdot & \cdot & \cdot & \cdot
\end{array}\right)
$$


if we use the lexicographic order $000,001,010,011,100,101,110,111$. It was shown [19] that the map $\phi$ is indecomposable positive bi-linear map. In fact, it was shown that $\phi$ has the full spanning property. See also [13] for indecomposability.

We note that all the entries of $C_{\phi}$ are zero except for diagonal and anti-diagonals. Such matrices are called $\mathrm{X}$-shaped, and are of the form

$$
X(a, b, c)=\left(\begin{array}{ccccccccc}
a_{1} & & & & & & & c_{1} \\
& a_{2} & & & & & c_{2} & \\
& & a_{3} & & & c_{3} & & \\
& & & a_{4} & c_{4} & & & \\
& & & \bar{c}_{4} & b_{4} & & & \\
& & \bar{c}_{3} & & & b_{3} & & \\
& \bar{c}_{2} & & & & & b_{2} & \\
\bar{c}_{1} & & & & & & & b_{1}
\end{array}\right),
$$

for vectors $a=\left(a_{1}, a_{2}, a_{3}, a_{4}\right), b=\left(b_{1}, b_{2}, b_{3}, b_{4}\right) \in \mathbb{R}^{4}$ and $c=\left(c_{1}, c_{2}, c_{3}, c_{4}\right) \in \mathbb{C}^{4}$. Many important three qubit states arise in this form. For example, GreenbergerHorne-Zeilinger diagonal states are $\mathbf{X}$-shaped, and $\varrho=X(a, b, c)$ is GHZ diagonal if and only if $a=b$ and $c \in \mathbb{R}^{4}$.

\section{EXPOSEDNESS}

We first find the dual face of $\phi$. To do this, we look for pure product states $\varrho=|\xi\rangle\langle\xi|$ with the product vector $|\xi\rangle=|x\rangle \otimes|y\rangle \otimes|z\rangle$ satisfying the relation

$$
0=\langle\varrho, \phi\rangle=\left\langle C_{\phi}, \mid \xi\right\rangle\langle\xi \mid\rangle=\left\langle\bar{\xi}\left|C_{\phi}\right| \bar{\xi}\right\rangle=\langle\bar{z}|\phi(|x\rangle\langle x|,| y\rangle\langle y|)| \bar{z}\rangle .
$$

We first consider the case when one of $|x\rangle,|y\rangle$ or $|z\rangle$ has a zero entry. In this case, it is easy to see [19] that product vectors satisfying the relation (3) are one of the following:

$$
\begin{array}{rlrl}
|x\rangle 0\rangle 1\rangle=x_{0}|001\rangle+x_{1}|101\rangle, & & |x\rangle 1\rangle 0\rangle=x_{0}|010\rangle+x_{1}|110\rangle, \\
|0\rangle y\rangle 0\rangle=y_{0}|000\rangle+y_{1}|010\rangle, & & |1\rangle y\rangle 1\rangle=y_{0}|101\rangle+y_{1}|111\rangle, \\
|0\rangle 0\rangle z\rangle=z_{0}|000\rangle+z_{1}|001\rangle, & |1\rangle 1\rangle z\rangle=z_{0}|110\rangle+z_{1}|111\rangle,
\end{array}
$$

with $|x\rangle,|y\rangle,|z\rangle \in \mathbb{C}^{2}$.

Next, we consider the case when $|\xi\rangle$ has no zero entry. In this case, the X-part $\varrho_{X}$ of $\varrho=|\xi\rangle\langle\xi|$ is again a separable state of rank four. In fact, it was shown in [15] that $\varrho_{X}$ is the average $\varrho_{X}=\frac{1}{4} \sum_{k=0}^{3}|\xi(k)\rangle\langle\xi(k)|$ of the four pure product states given by

$$
\begin{aligned}
& |\xi(0)\rangle=\left|x_{+}\right\rangle \otimes\left|y_{+}\right\rangle \otimes\left|z_{+}\right\rangle, \\
& |\xi(1)\rangle=\left|x_{+}\right\rangle \otimes\left|y_{-}\right\rangle \otimes\left|z_{-}\right\rangle, \\
& |\xi(2)\rangle=\left|x_{-}\right\rangle \otimes\left|y_{+}\right\rangle \otimes\left|z_{-}\right\rangle, \\
& |\xi(3)\rangle=\left|x_{-}\right\rangle \otimes\left|y_{-}\right\rangle \otimes\left|z_{+}\right\rangle,
\end{aligned}
$$

with $\left|x_{ \pm}\right\rangle=\left(x_{0}, \pm x_{1}\right)^{\mathrm{t}}$ and $\left|y_{ \pm}\right\rangle,\left|z_{ \pm}\right\rangle$similarly. Furthermore, every rank four separable non-diagonal $\mathrm{X}$-state arises in this way with a unique decomposition. 
Because $C_{\phi}$ is $\mathrm{X}$-shaped, the relation (3) is equivalent to $\left\langle C_{\phi}, \varrho_{X}\right\rangle=0$. Therefore, we proceed to find non-diagonal X-state $\varrho=X(a, b, c)$ of rank four so that $\langle\phi, \varrho\rangle=0$. Note [15] that $\varrho=X(a, b, c)$ is non-diagonal separable state of rank four if and only if

$$
a_{i} b_{i}=\left|c_{j}\right|^{2}(i, j=1,2,3,4), \quad a_{1} a_{4}=a_{2} a_{3}, \quad c_{1} c_{4}=c_{2} c_{3}
$$

We may assume that $a_{i} b_{i}=\left|c_{j}\right|=1$. Since $\left\langle C_{\phi}, \varrho\right\rangle=t a_{4}+s b_{4}+2 \operatorname{Re}\left(c_{1}+c_{2}-c_{3}+c_{4}\right)$, we have

$$
2 \sqrt{2}=\sqrt{s t} \leq \frac{1}{2}\left(t a_{4}+s b_{4}\right)=-\operatorname{Re}\left(c_{1}+c_{2}-c_{3}+c_{4}\right)
$$

Putting $\bar{c}_{1} c_{2}=\bar{c}_{3} c_{4}=-e^{\mathrm{i} \theta}$, we also have

$$
\begin{aligned}
2 \sqrt{2} \leq-\operatorname{Re}\left(c_{1}+c_{2}-c_{3}+c_{4}\right) & \leq\left|c_{1}+c_{2}\right|+\left|c_{3}-c_{4}\right| \\
& =\left|1-e^{\mathrm{i} \theta}\right|+\left|1+e^{\mathrm{i} \theta}\right| \leq 2 \sqrt{2} .
\end{aligned}
$$

Therefore, all the inequality in (6) and (7) becomes the equalities to get

- $t a_{4}=s b_{4}$ which implies $a_{4}=\sqrt{s / t}$,

- both $c_{1}+c_{2}$ and $-c_{3}+c_{4}$ are non-positive real numbers,

- $\theta=\frac{\pi}{2}$ or $\theta=-\frac{\pi}{2}$.

In the case of $\theta=\frac{\pi}{2}$, we see that both $c_{1}+c_{2}=c_{1}\left(1-e^{\mathrm{i} \frac{\pi}{2}}\right)$ and $-c_{3}+c_{4}=-c_{3}\left(1+e^{\mathrm{i} \frac{\pi}{2}}\right)$ are non-positive real numbers, and so we have $c=\left(\omega^{-3}, \omega^{3}, \omega^{-1}, \omega^{-3}\right)$, where $\omega=e^{\mathrm{i} \frac{\pi}{4}}$ is the 8 -th root of unity. If $\theta=-\frac{\pi}{2}$ then we also have $c=\left(\omega^{3}, \omega^{-3}, \omega, \omega^{3}\right)$. So, we have two kinds of rank four separable states

$$
\begin{aligned}
& \varrho_{1}\left(a_{1}, a_{2}\right)=X\left(\left(a_{1}, a_{2}, u a_{1} a_{2}^{-1}, u\right),\left(a_{1}^{-1}, a_{2}^{-1}, u^{-1} a_{1}^{-1} a_{2}, u^{-1}\right),\left(\omega^{-3}, \omega^{3}, \omega^{-1}, \omega^{-3}\right)\right), \\
& \varrho_{2}\left(a_{1}, a_{2}\right)=X\left(\left(a_{1}, a_{2}, u a_{1} a_{2}^{-1}, u\right),\left(a_{1}^{-1}, a_{2}^{-1}, u^{-1} a_{1}^{-1} a_{2}, u^{-1}\right),\left(\omega^{3}, \omega^{-3}, \omega, \omega^{3}\right)\right)
\end{aligned}
$$

in the dual $\phi^{\prime}$ of $\phi$, with $u:=\sqrt{\frac{s}{t}}$ and $a_{1}, a_{2}>0$. This is enough to compute the double dual $\phi^{\prime \prime}$.

For the completeness, we find product vectors

$$
\begin{aligned}
|\xi\rangle & =\left(p_{1}, \alpha_{1}\right)^{\mathrm{t}} \otimes\left(p_{2}, \alpha_{2}\right)^{\mathrm{t}} \otimes\left(p_{3}, \alpha_{3}\right)^{\mathrm{t}} \\
& =\left(p_{1} p_{2} p_{3}, p_{1} p_{2} \alpha_{3}, p_{1} \alpha_{2} p_{3}, p_{1} \alpha_{2} \alpha_{3}, \alpha_{1} p_{2} p_{3}, \alpha_{1} p_{2} \alpha_{3}, \alpha_{1} \alpha_{2} p_{3}, \alpha_{1} \alpha_{2} \alpha_{3}\right)^{\mathrm{t}}
\end{aligned}
$$

with $p_{i}>0$ and $\alpha_{i} \in \mathbb{T}$ satisfying the relation $r \varrho_{1}=|\xi\rangle\left\langle\left.\xi\right|_{X}\right.$ with $r>0$. Taking the $\mathrm{X}$-part, we have

$$
\begin{aligned}
r\left(a_{1}, a_{2}, u a_{1} a_{2}^{-1}, u\right) & =\left(p_{1}^{2} p_{2}^{2} p_{3}^{2}, p_{1}^{2} p_{2}^{2}, p_{1}^{2} p_{3}^{2}, p_{1}^{2}\right), \\
r\left(a_{1}^{-1}, a_{2}^{-1}, u^{-1} a_{1}^{-1} a_{2}, u^{-1}\right) & =\left(1, p_{3}^{2}, p_{2}^{2}, p_{2}^{2} p_{3}^{2}\right), \\
r\left(\omega^{-3}, \omega^{3}, \omega^{-1}, \omega^{-3}\right) & =p_{1} p_{2} p_{3}\left(\bar{\alpha}_{1} \bar{\alpha}_{2} \bar{\alpha}_{3}, \bar{\alpha}_{1} \bar{\alpha}_{2} \alpha_{3}, \bar{\alpha}_{1} \alpha_{2} \bar{\alpha}_{3}, \bar{\alpha}_{1} \alpha_{2} \alpha_{3}\right),
\end{aligned}
$$

which implies $r=p_{1} p_{2} p_{3}=a_{1}$ together with

$$
\alpha_{1}^{2}=\omega^{6}, \quad \alpha_{2}^{2}=\omega^{2}, \quad \alpha_{3}^{2}=\omega^{6}, \quad p_{6}^{2}=u a_{1}, \quad p_{2}^{2}=u^{-1} a_{2}, \quad p_{3}^{2}=a_{1} a_{2}^{-1} .
$$


Therefore, $\left(\alpha_{1}, \alpha_{2}\right)$ is one of $\left(\omega^{3}, \omega\right),\left(\omega^{3}, \omega^{5}\right),\left(\omega^{7}, \omega\right)$ and $\left(\omega^{7}, \omega^{5}\right)$. In each case, $\alpha_{3}$ is automatically determined. In this way, we have the following four kinds

$$
\begin{aligned}
& \eta_{1}\left(a_{1}, a_{2}\right)=\left(u^{\frac{1}{2}} a_{1}^{\frac{1}{2}}, \omega^{3}\right)^{\mathrm{t}} \otimes\left(u^{-\frac{1}{2}} a_{2}^{\frac{1}{2}}, \omega\right)^{\mathrm{t}} \otimes\left(a_{1}^{\frac{1}{2}} a_{2}^{-\frac{1}{2}}, \omega^{7}\right)^{\mathrm{t}}, \\
& \eta_{2}\left(a_{1}, a_{2}\right)=\left(u^{\frac{1}{2}} a_{1}^{\frac{1}{2}}, \omega^{3}\right)^{\mathrm{t}} \otimes\left(u^{-\frac{1}{2}} a_{2}^{\frac{1}{2}}, \omega^{5}\right)^{\mathrm{t}} \otimes\left(a_{1}^{\frac{1}{2}} a_{2}^{-\frac{1}{2}}, \omega^{3}\right)^{\mathrm{t}}, \\
& \eta_{3}\left(a_{1}, a_{2}\right)=\left(u^{\frac{1}{2}} a_{1}^{\frac{1}{2}}, \omega^{7}\right)^{\mathrm{t}} \otimes\left(u^{-\frac{1}{2}} a_{2}^{\frac{1}{2}}, \omega\right)^{\mathrm{t}} \otimes\left(a_{1}^{\frac{1}{2}} a_{2}^{-\frac{1}{2}}, \omega^{3}\right)^{\mathrm{t}}, \\
& \eta_{4}\left(a_{1}, a_{2}\right)=\left(u^{\frac{1}{2}} a_{1}^{\frac{1}{2}}, \omega^{7}\right)^{\mathrm{t}} \otimes\left(u^{-\frac{1}{2}} a_{2}^{\frac{1}{2}}, \omega^{5}\right)^{\mathrm{t}} \otimes\left(a_{1}^{\frac{1}{2}} a_{2}^{-\frac{1}{2}}, \omega^{7}\right)^{\mathrm{t}}
\end{aligned}
$$

of product vectors satisfying (3), up to constant multiples. In case of $\theta=-\frac{\pi}{2}$, we consider the relation $r \varrho_{2}=|\xi\rangle\left\langle\left.\xi\right|_{X}\right.$ to get the following four product vectors

$$
\begin{aligned}
& \zeta_{1}\left(a_{1}, a_{2}\right)=\left(u^{\frac{1}{2}} a_{1}^{\frac{1}{2}}, \omega^{5}\right)^{\mathrm{t}} \otimes\left(u^{-\frac{1}{2}} a_{2}^{\frac{1}{2}}, \omega^{7}\right)^{\mathrm{t}} \otimes\left(a_{1}^{\frac{1}{2}} a_{2}^{-\frac{1}{2}}, \omega\right)^{\mathrm{t}}, \\
& \zeta_{2}\left(a_{1}, a_{2}\right)=\left(u^{\frac{1}{2}} a_{1}^{\frac{1}{2}}, \omega^{5}\right)^{\mathrm{t}} \otimes\left(u^{-\frac{1}{2}} a_{2}^{\frac{1}{2}}, \omega^{3}\right)^{\mathrm{t}} \otimes\left(a_{1}^{\frac{1}{2}} a_{2}^{-\frac{1}{2}}, \omega^{5}\right)^{\mathrm{t}}, \\
& \zeta_{3}\left(a_{1}, a_{2}\right)=\left(u^{\frac{1}{2}} a_{1}^{\frac{1}{2}}, \omega\right)^{\mathrm{t}} \otimes\left(u^{-\frac{1}{2}} a_{2}^{\frac{1}{2}}, \omega^{7}\right)^{\mathrm{t}} \otimes\left(a_{1}^{\frac{1}{2}} a_{2}^{-\frac{1}{2}}, \omega^{5}\right)^{\mathrm{t}}, \\
& \zeta_{4}\left(a_{1}, a_{2}\right)=\left(u^{\frac{1}{2}} a_{1}^{\frac{1}{2}}, \omega\right)^{\mathrm{t}} \otimes\left(u^{-\frac{1}{2}} a_{2}^{\frac{1}{2}}, \omega^{3}\right)^{\mathrm{t}} \otimes\left(a_{1}^{\frac{1}{2}} a_{2}^{-\frac{1}{2}}, \omega\right)^{\mathrm{t}} .
\end{aligned}
$$

In total, we found all the product vectors satisfying (3) listed in (44), (8) and (9). We note that four product vectors $\eta_{1}, \eta_{4}, \zeta_{1}, \zeta_{4}$ were missing in [19], and correct here.

Now, we proceed to find the double dual dual $\phi^{\prime \prime}$ of $\phi$. In other word, we are looking for self-adjoint block-positive three qubit matrices $W$ satisfying the relation $\langle W, \varrho\rangle=0$ for every $\varrho \in \phi^{\prime}$. We see from the list (4) that $\phi^{\prime}$ contains the pure product state associated with the following product vectors:

$$
|0\rangle 0\rangle 0\rangle, \quad|0\rangle 0\rangle 1\rangle, \quad|0\rangle 1\rangle 0\rangle, \quad|1\rangle 0\rangle 1\rangle, \quad|1\rangle 1\rangle 0\rangle, \quad|1\rangle 1\rangle 1\rangle .
$$

Therefore, the corresponding diagonal entries of $W$ must be zero. From this, we show that $W$ must be $\mathrm{X}$-shaped. We note that the $4 \times 4$ principle submatrix $W_{0}$ of $W$ with entries from $\{000,001,010,011\}$ is block-positive with zero diagonals for $000,001,010$ entries. Because the $2 \times 2$ principle submatrices with entries $\{000,001\},\{000,010\}$ $\{010,011\},\{001,011\}$ are positive, we see that $W_{0}$ is of the form

$$
W_{0}=\left(\begin{array}{cccc}
\cdot & \cdot & \cdot & u \\
\cdot & \cdot & v & \cdot \\
\cdot & \bar{v} & \cdot & \cdot \\
\bar{u} & \cdot & \cdot & 1
\end{array}\right)
$$

up to scalar multiplication. We note that the corresponding positive map sends the positive matrix $P_{\alpha}:=\left(\begin{array}{cc}1 & \alpha \\ \bar{\alpha} & |\alpha|^{2}\end{array}\right)$ to the $2 \times 2$ matrix $\left(\begin{array}{cc}0 & \alpha u+\bar{\alpha} \bar{v} \\ \bar{\alpha} \bar{u}+\alpha v & |\alpha|^{2}\end{array}\right)$ which must be positive. This implies that $\alpha u+\bar{\alpha} \bar{v}=0$ for every complex $\alpha$, from which we conclude that $u=v=0$. Therefore, all the entries of $W_{0}$ are zero except for 011 place. In the above argument, we fix 0 in the first place of indices to get $4 \times 4$ principle submatrix which is still block-positive. We fix 0 or 1 in the first, second or third places to get six $4 \times 4$ principle submatrices which are still block-positive. All the entries of such 
principle submatrices are zero except for diagonal entries at 011 and 100 places. This implies that $W$ must be $\mathrm{X}$-shaped.

Now, we can write $W=X\left(\left(0,0,0, x_{4}\right),\left(0,0,0, y_{4}\right),\left(z_{1}, z_{2}, z_{3}, z_{4}\right)\right)$. By [12], we know that $W$ is block-positive if and only if

$$
\sqrt{x_{4} y_{4}} \geq \max _{\theta}\left(\left|z_{1} e^{\mathrm{i} \theta}+\bar{z}_{4}\right|+\left|z_{2} e^{\mathrm{i} \theta}+\bar{z}_{3}\right|\right):=\|z\|_{\mathrm{x}}
$$

See [1] for properties of the norm \|\|$_{x}$ defined as above. Considering the state

$$
\varrho:=\frac{1}{2}\left(\varrho_{1}(1,1)+\varrho_{2}(1,1)\right)=X\left((1,1, u, u),\left(1,1, u^{-1}, u^{-1}\right), \frac{1}{\sqrt{2}}(-1,-1,1,-1)\right)
$$

in the dual $\phi^{\prime}$, we have

$$
0=\langle\varrho, W\rangle=x_{4} u+y_{4} u^{-1}-\sqrt{2} \operatorname{Re}\left(z_{1}+z_{2}-z_{3}+z_{4}\right)
$$

Therefore, we have

$$
\begin{aligned}
2 \sqrt{2}\|z\|_{\mathrm{x}} & \leq \sqrt{2} \cdot 2 \sqrt{x_{4} y_{4}} \\
& \leq \sqrt{2}\left(x_{4} \sqrt{s / t}+y_{4} \sqrt{t / s}\right) \\
& =2 \operatorname{Re}\left(z_{1}+z_{2}-z_{3}+z_{4}\right) \\
& \leq 2\left(\left|z_{1}\right|+\left|z_{2}\right|+\left|z_{3}\right|+\left|z_{4}\right|\right) \leq 2 \sqrt{2}\|z\|_{\mathrm{x}} .
\end{aligned}
$$

See [1] and Appendix of [14] for the last inequality $\|z\|_{\mathrm{x}} \geq \frac{1}{\sqrt{2}}\|z\|_{1}$, where the equality holds only if the entries have a common magnitude and

$$
\left(\arg z_{1}+\arg z_{4}\right)-\left(\arg z_{2}+\arg z_{3}\right)=\pi
$$

by Proposition 3.8 and (27) in [1]. Therefore, we have the following:

(i) $\sqrt{2}\|z\|_{\mathrm{x}}=\|z\|_{1}$, and so $z$ has a common magnitude,

(ii) $x_{4} \sqrt{s / t}=y_{4} \sqrt{t / s} \Longleftrightarrow x_{4} s=y_{4} t$,

(iii) $\operatorname{Re}\left(z_{1}+z_{2}-z_{3}+z_{4}\right)=\left|z_{1}\right|+\left|z_{2}\right|+\left|z_{3}\right|+\left|z_{4}\right|$.

From (i) and (iii), we see that $z=r_{1}(1,1,-1,1)$ for a nonnegative $r_{1}>0$, and we also have $\left(x_{4}, y_{4}\right)=r_{2}(t, s)$ for $r_{2}>0$ by (ii). Then we have $2 \operatorname{Re}\left(z_{1}+z_{2}-z_{3}+z_{4}\right)=8 r_{1}$ and $\sqrt{2}\left(x_{4} \sqrt{s / t}+y_{4} \sqrt{t / s}\right)=\sqrt{2} \cdot 2 \sqrt{s t} \cdot r_{2}=8 r_{2}$. Therefore, we have $r_{1}=r_{2}$ by the equality in (11), and finally conclude that $W$ is a nonnegative multiple of $C_{\phi}$. This completes the proof that the bi-linear map $\phi$ is exposed.

\section{REFERENCES}

[1] L. Chen, K. H. Han and S.-H. Kye, Separability criterion for three-qubit states with a four dimensional norm, J. Phys. A: Math. Theor. 50 (2017) 345303.

[2] S.-J. Cho, S.-H. Kye and S. G. Lee, Generalized Choi maps in 3-dimensional matrix algebras, Linear Alg. Appl. 171 (1992), 213-224.

[3] M.-D. Choi, Completely positive linear maps on complex matrices, Linear Alg. Appl. 10 (1975), 285-290.

[4] M.-D. Choi, Positive semidefinite biquadratic forms, Linear Alg. Appl. 12 (1975), 95-100.

[5] M.-D. Choi and T.-T. Lam, Extremal positive semidefinite forms, Math. Ann. 231 (1977), 1-18.

[6] D. Chruściński and G. Sarbicki, Spectral Conditions for Positive Maps, Commun. Math. Phys. 290 (2009), 1051-1064. 
[7] D. Chruściński and G. Sarbicki, Exposed positive maps in $M_{4}(\mathbb{C})$, Open Sys. Inf. Dyn. 19 (2012), 1250017.

[8] M.-H. Eom and S.-H. Kye, Duality for positive linear maps in matrix algebras, Math. Scand. 86 (2000), 130-142.

[9] K.-C. Ha and S.-H. Kye, Exposedness of Choi type entanglement witnesses and applications to lengths of separable states, Open Syst. Inf. Dyn. 20 (2013), 1350012.

[10] K.-C. Ha and S.-H. Kye, Separable states with unique decompositions, Commun. Math. Phys. 328 (2014), 131-153.

[11] K.-C. Ha and S.-H. Kye, Construction of exposed indecomposable positive linear maps between matrix algebras, Linear Multilinear Alg. 64 (2016), 2188-2198.

[12] K. H. Han and S.-H, Kye, Various notions of positivity for bi-linear maps and applications to tri-partite entanglement, J. Math. Phys. 57 (2016), 015205.

[13] K. H. Han and S.-H, Kye, Construction of multi-qubit optimal genuine entanglement witnesses, J. Phys. A: Math. Theor. 49 (2016), 175303.

[14] K. H. Han and S.-H, Kye, Separability of three qubit Greenberger-Horne-Zeilinger diagonal states, J. Phys. A: Math. Theor. 50 (2017), 145303.

[15] K. H. Han and S.-H, Kye, The role of phases in detecting three qubit entanglement, preprint, arXiv:1610.06645.

[16] M. Horodecki, P. Horodecki and R. Horodecki, Separability of mixed states: necessary and sufficient conditions, Phys. Lett. A 223 (1996), 1-8.

[17] A. Jamiołkowski, An effective method of investigation of positive maps on the set of positive definite operators, Rep. Math. Phys. 5 (1974), 415-424.

[18] S.-H. Kye, Facial structures for various notions of positivity and applications to the theory of entanglement, Rev. Math. Phys. 25 (2013), 1330002.

[19] S.-H. Kye, Three-qubit entanglement witnesses with the full spanning properties J. Phys. A: Math. Theor. 48 (2015), 235303.

[20] M. Marciniak, Rank properties of exposed positive maps, Linear Multilinear Alg. 61 (2013), 970975.

[21] A. G. Robertson, Automorphisms of spin factors and the decomposition of positive maps, Quart. J. Math. Oxford 34 (1983), 87-96.

[22] A. G. Robertson, Positive projections on $C^{*}$-algebras and extremal positive maps, J. London Math. Soc. 32 (1985), 133-140.

[23] R. T. Rockafellar, Convex Analysis, Princeton University Press, 1970.

[24] G. Sarbicki and D. Chruściński, A class of exposed indecomposable positive maps J. Phys. A: Math. Theor. 46 (2013) 015306.

[25] E. Størmer, Positive linear maps of operator algebras, Acta Math. 110 (1963), 233-278.

[26] S. L. Woronowicz, Positive maps of low dimensional matrix algebras, Rep. Math. Phys. 10 (1976), $165-183$.

[27] S. L. Woronowicz, Nonextendible positive maps, Commun. Math. Phys. 51 (1976), 243-282.

[28] D. A. Yopp and R. D. Hill, Extremals and exposed faces of the cone of positive maps, Linear and Multilinear Alg. 53 (2005), 167-174.

Department of Mathematics and Institute of Mathematics, Seoul National UniVERSiTy, SEOUl 151-742, KOREA

E-mail address: kye at snu.ac.kr 